\title{
石墨烯修饰的柔性多彩电热致变色薄膜的制备及性能研究
}

\author{
张 斌 ${ }^{1}$, 侯成义 ${ }^{2}$, 汪浩鹏 ${ }^{1}$, 汪志强 ${ }^{1}$, 柏宇苗 ${ }^{2}$, \\ 李 强 ${ }^{2}$, 张青红 ${ }^{2}$, 李耀刚 ${ }^{2}$, 王宏志 ${ }^{2}$
}

(1. 中国电子科技集团公司信息科学研究院 微系统技术研究所, 北京 100086; 2. 东华大学 材料科学与工程学院, 纤维材料改性国家重点实验室, 上海 201620)

摘 要: 通过化学氧化还原法并辅以抽滤法制备 RGO 导电薄膜, 通过丝网印刷法制备变色层, 构筑了多层结构的多 彩(红-蓝-白以及橙红-黄-白等)柔性电热致变色薄膜。采用 SEM、XRD 以及 Raman 等分析薄膜的结构性质。采用 红外热成像以及吸收光谱研究了薄膜(红一蓝-白)的热学以及变色性能。结果表明: 当加热时间为 $3.4 \mathrm{~s}$ 时, 薄膜温 度能达到 $38^{\circ} \mathrm{C}$, 变为蓝色; 当加热时间为 $6.3 \mathrm{~s}$ 时, 薄膜温度达到 $45^{\circ} \mathrm{C}$, 变为白色。在较低电压下 $(6 \mathrm{~V})$, 该薄膜能 实现多色彩可逆变色, 其变色时间约为 $6.3 \mathrm{~s}$, 褪色时间约为 $9.2 \mathrm{~s}$ 。该柔性电热致变色薄膜以混合纤维素滤膜为基 体，保证了薄膜良好的柔性，在可穿戴显示领域有着一定的应用价值。

关 键 词: 石墨烯; 柔性; 多彩; 电热致变色

中图分类号: TQ174 文献标识码: A

\section{Preparation and Performance of Reduced Graphene Oxide Functionalized Flexible and Multicolor Electrothermal Chromatic Films}

\author{
ZHANG Bin ${ }^{1}$, HOU Cheng-Yi ${ }^{2}$, WANG Hao-Peng ${ }^{1}$, WANG Zhi-Qiang ${ }^{1}$, BAI Yu-Miao ${ }^{2}$, \\ LI Qiang ${ }^{2}$, ZHANG Qing-Hong ${ }^{2}$, LI Yao-Gang ${ }^{2}$, WANG Hong-Zhi ${ }^{2}$
}

(1. Microsystems Technology Center, Information Science Academy of China Electronics Technology Group Corporation, Beijing 100086; 2. State Key Laboratory for Modification of Chemical Fibers and Polymer Materials, College of Materials Science and Engineering, Donghua University, Shanghai 201620, China)

\begin{abstract}
By using a chemical oxidation-reduction method, reduced graphene oxide (RGO) nanomaterials were synthesized. The powder materials were further dispersed in water and filtered to form a RGO film, which was used as a conductive layer. On the other side of the filter paper, a color changing layer was prepared through screen printing. A multicolor (red-blue-white and orange-yellow-white, etc.) and flexible electrothermal chromatic film was then obtained through combining conductive and color changing layers with protecting substrates. The structural properties of the film (red-blue-white) were analyzed by using SEM, XRD and Raman, etc. Their thermal and color-changing properties were studied by using infrared thermal imaging and absorption spectrum. The results show that when surface temperature of the film reaches $38^{\circ} \mathrm{C}$ (within $3.4 \mathrm{~s}$ under Joule heat treatment at ambient conditions), it turns blue. Further heating to $45^{\circ} \mathrm{C}$ (within $6.3 \mathrm{~s}$ under Joule heat treatment), it turns white. The multi-color changes are achieved at low working voltage $(6 \mathrm{~V})$. After switching off the voltage, the film restores its original color within $9.2 \mathrm{~s}$. The electrothermal chromatic films have good flexibility too, therefore, they might be applied in wearable displays.
\end{abstract}

收稿日期: 2018-01-31; 收到修改稿日期：2018-04-20

基金项目：上海市晨光计划(15CG33); 上海市扬帆计划(16YF1400400)

Shanghai ChenGuang Program (15CG33); Shanghai Sailing Program (16YF1400400)

作者简介: 张斌(1980-), 男, 高级工程师. E-mail: ivan_bin@126.com

通讯作者: 侯成义, 讲师. E-mail: hcy@dhu.edu.cn; 李强, 工程师. E-mail:18306481933@163.com 
Key words: RGO; flexible; multicolor; electrothermal chromatic

近年来，随着可穿戴技术的不断发展，柔性智 能变色器件已成为当前的研究热点, 并有望广泛地 应用在军事伪装、可穿戴式显示和可视化传感等领 域 ${ }^{[1-4]}$ 。其中, 柔性电热致变色器件因结构简单、可 控性强而备受青睐 ${ }^{[5-8]}$ 。

但是，由于制备具有良好导电性和稳定性的柔 性导电器件非常困难, 柔性电热致变色薄膜没有真 正实现市场化。其中导电材料是实现良好性能的关 键因素之一, 但传统的导电材料(金属或合金)已无法 满足柔性可穿戴设备的要求。尽管导电聚合物 ${ }^{[9-10]}$ 、 金属纳米线 ${ }^{[11-12]}$ 等新型导电材料可与柔性基体进行 良好的结合，但导电聚合物的老化问题和金属纳米 线的氧化问题又限制了它们的应用范畴。而石墨烯 (RGO) 不仅导电性和化学稳定性良好, 而且可设计 性强, 成为备受青睐的柔性薄膜导电材料 ${ }^{[13-18]}$ 。

本工作通过化学氧化还原法制备 RGO 薄膜并 将其作为导电层, 使用聚酰亚胺(PI) 作为保护层, 柔性混合纤维素滤膜作为基底层, 并通过丝网印刷 制备变色层, 最终获得多层结构的柔性电热致变色 薄膜, 并对变色薄膜的结构、电学、热学性能进行 了研究, 对薄膜的变色以及褪色时间进行了探讨。

\section{1 实验方法}

\section{1 还原氧化石墨烯原液的制备}

首先，采用改性 Hummers 法制备 GO 粉末 ${ }^{[19]}$ 。 将 $100 \mathrm{mg}$ 氧化石墨烯粉末分散到 $100 \mathrm{~mL}$ 去离子水 中, 并用细胞粉碎机超声 $2 \mathrm{~h}$ 。将 $4 \mathrm{~mL}$ 的氨水一次 性加入到 $\mathrm{GO}$ 中, 用 $\mathrm{pH}$ 计测量该混合液 $\mathrm{pH}$, 控制 在 9 12 内。量取 $90 \mu \mathrm{L}$ 氢碘酸溶液(HI)滴加氧化石 墨溶液中, 同时不断搅拌, 随后用加热台将混合液体 加热到 $90^{\circ} \mathrm{C}$, 在搅拌密封情况下保温 $2 \mathrm{~h}$, 溶液由棕 色变为黑色即完成氧化石墨还原过程, 得到还原氧 化石墨烯原液。

\section{2 柔性 RGO 薄膜的制备}

取 $30 \mathrm{~mL}$ 还原氧化石墨烯原液置于 $150 \mathrm{~mL}$ 烧 杯中, 再加入 $90 \mathrm{~mL}$ 超纯水稀释至 $100 \mathrm{~mL}$, 得到黑 色分散均匀的 RGO 分散液。利用混合纤维素载体 薄膜通过真空抽滤制备 $\mathrm{RGO} /$ 载体膜的复合薄膜, 将其置于 $60^{\circ} \mathrm{C}$ 真空烘箱中烘干 $4 \mathrm{~h}$, 得到表面光滑、 导电性好的 $\mathrm{RGO} /$ 载体膜的复合薄膜。

\section{3 柔性电热致变色薄膜的制备}

称取 $5 \mathrm{~g}$ 红色油墨与 $1 \mathrm{~g}$ 蓝色油墨 $(5: 1)$ 进行混 合, 并加 $3 \mathrm{~mL}$ 水稀释, 通过磁力搅拌器搅拌 $2 \mathrm{~h}$ 使 其混合均匀, 得到混合可逆热致变色油墨。将干燥 的复合薄膜反面放置于手工丝网印刷板下方, 取一 定量配制好的热致变色油墨置于印网上，通过刮板 将图案印刷至薄膜反面, 制备热致变色薄膜层, 得 到还原氧化石墨烯/载体薄膜/可逆热致变色薄膜组 成的 3 层结构复合薄膜, 并在 $60^{\circ} \mathrm{C}$ 真空烘箱烘干。 采用导电银线和导电银浆与导电铜胶带连接线路, 将 PI 胶带粘附在 RGO 表层制备保护层获得电热致 变色薄膜。

\section{4 测试方法}

采用 Phenom G2 型扫描电子显微镜(SEM)表征 样品的微观形貌; 采用 RigaKu D/max-2550Pc 型 X 射线衍射仪(XRD, 管压: $40 \mathrm{kV}$, 电流: $30 \mathrm{~mA}, \mathrm{CuK \alpha}$, $\lambda=0.154056 \mathrm{~nm}$ ) 对样品进行物相分析; 使用 Renishaw 激光拉曼仪表征样品的拉曼光谱; 使用 MCP-T3 60 型四探针电阻率测试仪测试样品的电导率/电阻率; 采用瑞典的 FLIR A300-Serious 型红外热成像仪和 FLIR ThermoVision A40 m 型红外测温仪表征样品 的热学性能; 使用复享光谱仪测试样品颜色的吸收 光谱。

\section{2 结果与分析}

\subsection{GO 与 RGO 的 XRD 及 Raman 分析}

图 1(a)为通过改性 Hummers 法制备的 GO 和 $\mathrm{HI}$ 还原得到的 RGO 的 XRD 图谱, 由图可知, $2 \theta=10.2^{\circ}$ 处衍射峰对应 GO 的(001)晶面的特征峰。 由 Bragg 方程计算得, 层间距 $d=0.867 \mathrm{~nm}$, 相比于 石墨 $d=0.335 \mathrm{~nm}$, 石墨片层间距增大, 表明石墨被 氧化。且在氧化过程中, 石墨片层上产生许多含氧 官能团。再通过 HI 还原之后, 位于 $(001)$ 晶面的衍射 峰消失, 衍射峰左移 $\left(2 \theta=23.8^{\circ}\right)$, 表明 $\mathrm{GO}$ 片层上的 含氧官能团消失, $\mathrm{GO}$ 被还原为 $\mathrm{RGO}$ 。

测试样品的 Raman 光谱并对其分析, 进一步研 究 $\mathrm{HI}$ 还原效果(图 1(b))。从图 1(b) 可知, GO 和 RGO 均在 1561 和 $1335 \mathrm{~cm}^{-1}$ 处出现 D 峰和 $\mathrm{G}$ 峰。在 HI 还原前后，二者的强度发生明显变化，这表明 $\mathrm{HI}$ 还 原改变了 $\mathrm{GO}$ 结构。计算 $\mathrm{D} 、 \mathrm{G}$ 峰强度之比 $\left(I_{\mathrm{D}} / I_{\mathrm{G}}\right)$, 还 
原前后分别为 0.92 和 1.49 , 说明结构缺陷数量的增 加以及 $\mathrm{sp}^{2}$ 杂化轨道的平均尺寸减小, 也能够证实 $\mathrm{GO}$ 在 $\mathrm{HI}$ 处理后还原较为充分 ${ }^{[14]}$ 。

\section{2 柔性电热致变色薄膜的性能研究}

\subsection{1 柔性电热致变色薄膜的结构分析}

图 2 为所制备的 RGO 修饰的柔性电热致变色 薄膜结构示意图, 薄膜由 4 层结构组成, 依次为保护 层、导电层、基底层以及变色层。保护层为耐热性 良好的聚酰亚胺(PI)胶带, 能够固定 RGO 的相对位 置, 防止 RGO 在使用过程中损坏甚至断裂; 基底层 为混合纤维素滤膜; 导电层为制备的 RGO 薄膜; 变 色层为手工丝网印刷的图案。多彩的柔性电热致变 色薄膜可以通过不同的热致变色油墨来实现。

柔性导电薄膜的多层结构可以从断面 SEM 照 片(图 3(a)) 中明显看出, 其厚度大概在 $100 \mu \mathrm{m}$, 为 三层结构: 最上面为导电层; 中间为约为 $50 \mu \mathrm{m}$ 滤 膜基体; 最下面是通过丝网印刷工艺印刷的变色 层。图 3(b)为一定弯曲程度的柔性导电薄膜的数码 照片。在制备保护层之后, 所制备的多层结构的电 热致变色薄膜仍具有一定的柔性。

为了便于观察薄膜的截面形貌, 制备了较厚的 RGO 样品。从截面 SEM 照片中(图 4)可知, RGO 薄 膜具有多层结构, 片层具有一定的可弯折性, 为器 件在弯曲条件下工作提供了保障。下文测试用柔性 电热致变色器件的 $\mathrm{RGO}$ 导电层厚度均为 $1 \mu \mathrm{m}$ 左右。
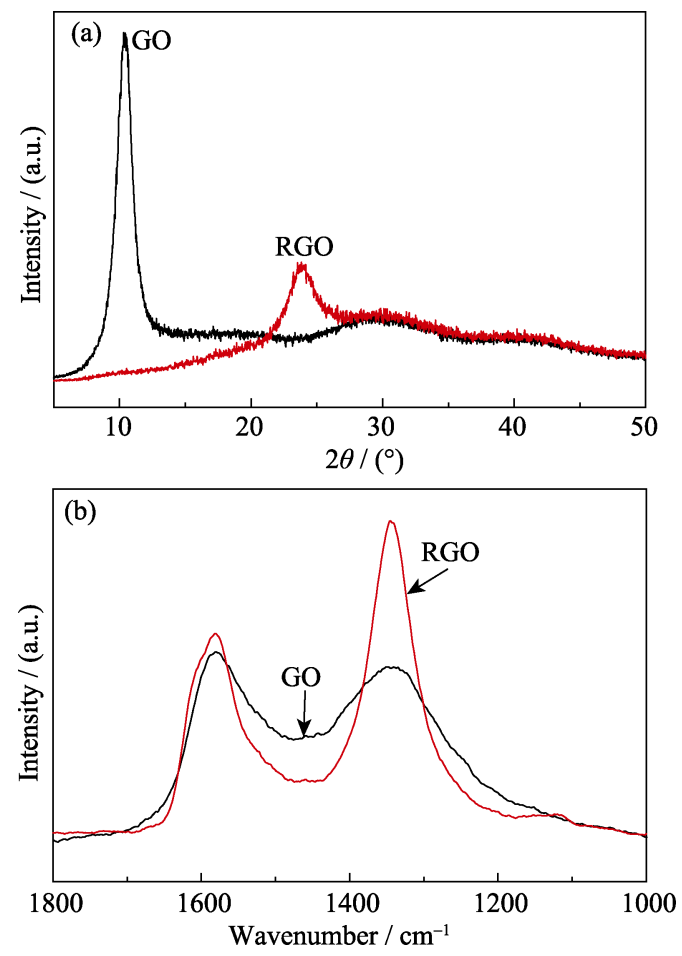

图 $1 \mathrm{GO}$ 与 $\mathrm{RGO}$ 的 XRD 图谱(a)以及拉曼光谱图(b)

Fig. 1 XRD patterns (a) and Raman spectra (b) of GO and RGO

\subsection{2 电热致变色薄膜的变色性能分析}

在氨气保护气氛下, 通过四探针法测试还原得 到的 RGO 薄膜的方阻为 $25.3 \Omega /$ 。在 $6 \mathrm{~V}$ 电压下, RGO 产生焦耳热, 热量传到变色层, 实现颜色的变 化。图 5(a) (e)为在通电下柔性电热致变色薄膜的数 码照片：在没通电时，器件呈现红色(图 5(a)); 通电 时, 当温度达到 $38^{\circ} \mathrm{C}$, 变色层中红色相消失, 故柔 性电热致变色薄膜显示蓝色相(图 5(b)); 随着温度 的进一步升高, 达到 $45^{\circ} \mathrm{C}$ 时, 变色层中蓝色相也随 之消失(图 5(c)); 当断开电源之后, 温度逐渐下降, 蓝色相和红色相依次显现出来(图 5(d) (e))。

在 $6 \mathrm{~V}$ 电压下, 柔性电热致变色薄膜能实现颜 色阶段性变化。由与之相对应的红外热成像(如图 6 所示)可见，随着通电时间的增加，其温度逐渐上 升。热量在薄膜分布得比较均匀, 表明通过抽滤法 制备的 RGO 薄膜具有良好的均匀性。
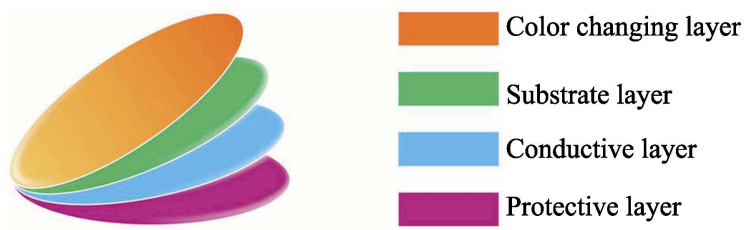

图 2 柔性电热致变色薄膜结构示意图

Fig. 2 Schematic illustration of the flexible electrothermal chromatic films
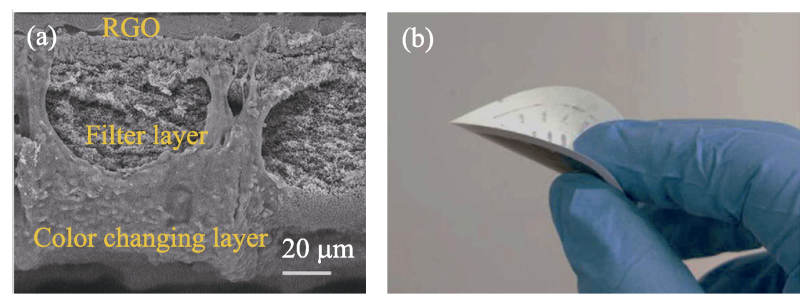

图 3 柔性导电薄膜的断面 SEM(a)及数码照片(b)

Fig. 3 Cross-sectional SEM image (a) and photograph (b) of the flexible conductive film

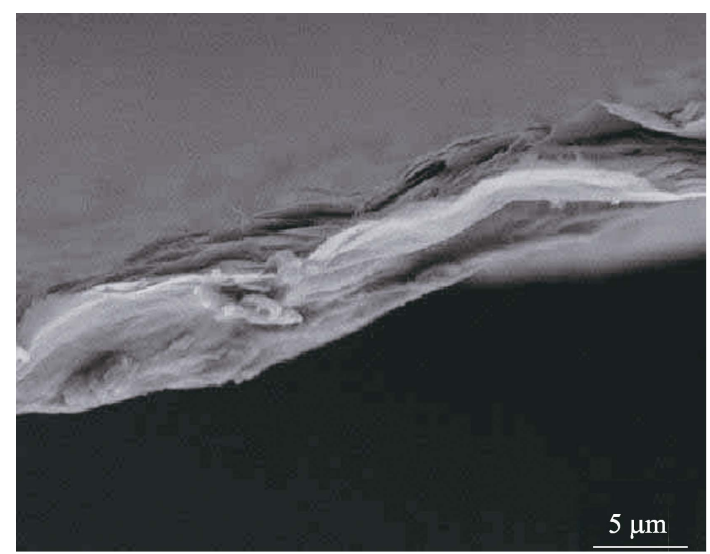

图 4 RGO 的 SEM 照片

Fig. 4 SEM image of RGO 

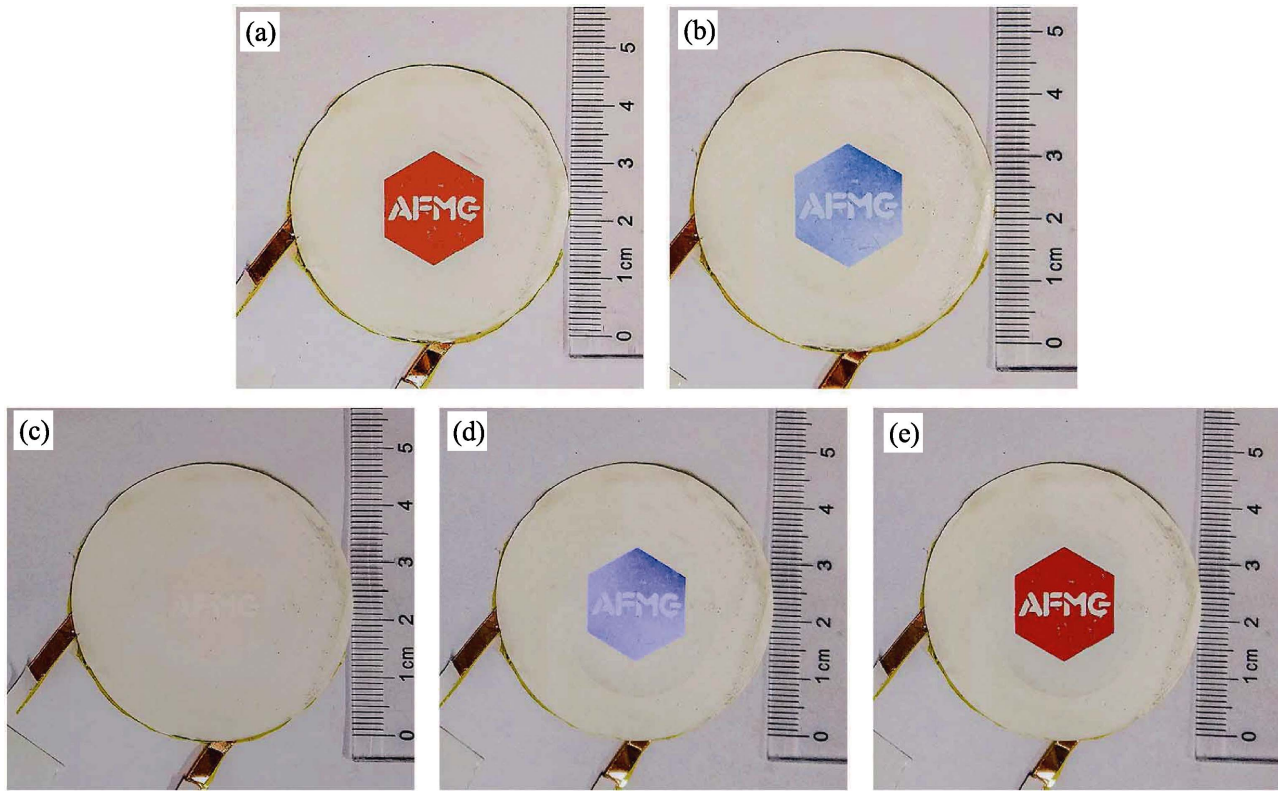

图 5 在 $6 \mathrm{~V}$ 电压下, 柔性电热致变色薄膜在一个周期内的颜色变化的数码照片

Fig. 5 Photographs of the flexible electrothermal chromatic film during a color-change cycle under an applied voltage of $6 \mathrm{~V}$
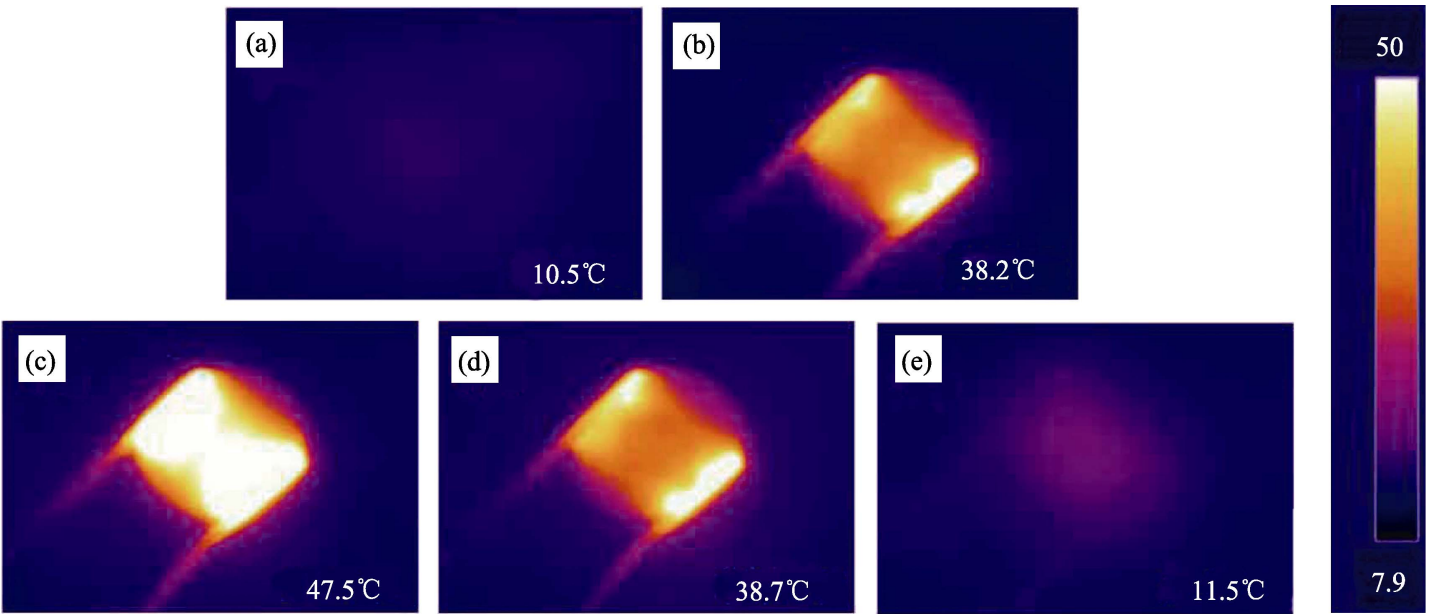

图 6 在 $6 \mathrm{~V}$ 电压下, 柔性电热致变色薄膜在一个周期内的红外热成像图

Fig. 6 Thermal images of the flexible electrothermal chromatic film during a cycle under an applied voltage of $6 \mathrm{~V}$

为了研究柔性电热致变色薄膜的热学性质, 对 器件在 $6 \mathrm{~V}$ 电压下, 温度与时间的关系进行表征分 析。所调制的混合变色油墨在 $38^{\circ} \mathrm{C}$ 以下为混合色, $38 \sim 45^{\circ} \mathrm{C}$ 范围为蓝色, $45^{\circ} \mathrm{C}$ 以上为无色。结合图 7 可 知, 当加热时间为 $3.4 \mathrm{~s}$ 时, 器件温度能达到 $38.2^{\circ} \mathrm{C}$; 当加热时间为 $6.3 \mathrm{~s}$ 时, 器件温度达到 $45.3^{\circ} \mathrm{C}$ 。因此, 制备的柔性电热致变色薄膜能够在较低的电压下实 现多种颜色的变化, 这也为探究薄膜的变色响应速 率提供了数据支持。

图 8(a)是柔性电热致变色薄膜处于红色、蓝色 和无色三种状态的吸收光谱曲线, 从光谱的变化可 以可以看出, 在红色态时器件对 350 700 $\mathrm{nm}$ 的光 均有吸收, 对于红色波段的光吸收较少, 显示红色; 在蓝色态时, 器件对于 450 500 $\mathrm{nm}$ 的光吸收最少,
而对于其它波段的光吸收较多，显示蓝色; 而在透 明态时，基本在整个可见光谱中都没有吸收，显示

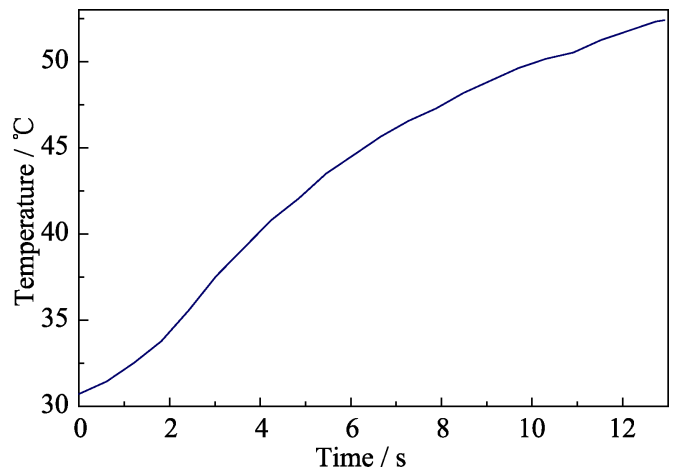

图 7 柔性电热致变色薄膜在 $6 \mathrm{~V}$ 电压下的温度时间曲线

Fig. 7 Temperature-time curves of the flexible electrothermal chromatic film under a current voltage of $6 \mathrm{~V}$ 

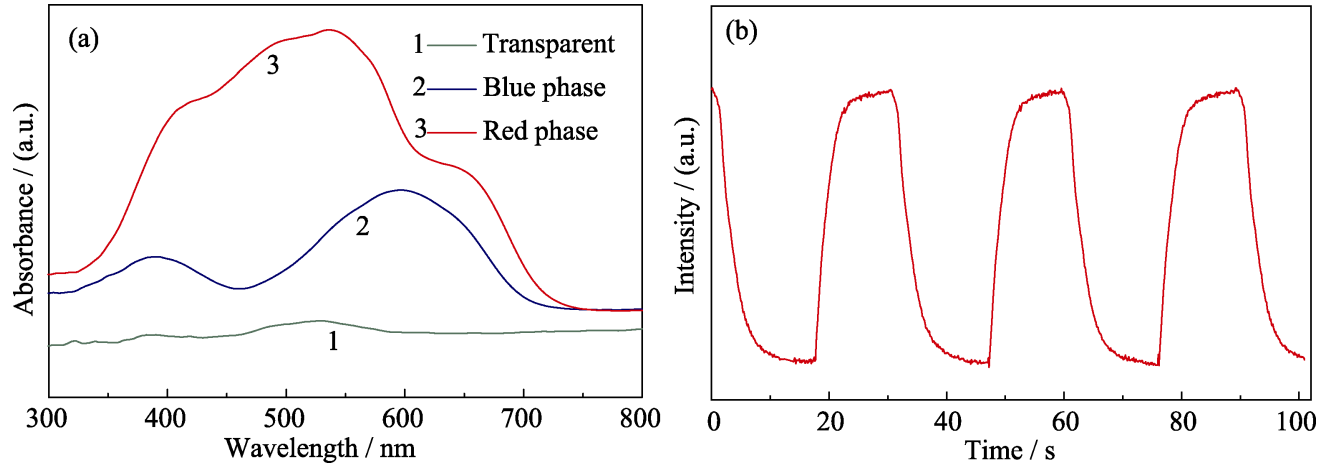

图 8 柔性电热致变色薄膜的吸收光谱(a)及响应时间(b)

Fig. 8 Absorbance spectra (a) and response time (b) of the flexible electrothermal chromatic film

无色, 也就是织物本身的颜色。图 8(b)给出了器件 的变色响应时间, 可以看出, 柔性电热致变色薄膜 的变色性能比较稳定, 响应时间基本不会发生变 化。这主要取决于 RGO 自身的化学稳定性, 不存在 金属纳米线的易氧化问题以及导电高分子的易老化 问题。另外, 对于变色阶段, 变色响应时间为 $6.3 \mathrm{~s}$, 与图 7 的温度时间曲线相符合; 对于褪色阶段, $9.2 \mathrm{~s}$ 恢复原来的颜色。柔性电热致变色薄膜具有较好的 柔性以及良好的变色性能、变色稳定性能, 可以满 足可穿戴显示的要求。

\section{3 结论}

通过氢碘酸还原法以及抽滤法制备出表面均 匀、导电性能良好的 RGO 薄膜, 将其作为导电层, 以 保证变色器件稳定的变色性能。通过设计多层薄膜 结构和选择变色油墨成功制备出 RGO 修饰的多色柔 性电热致变色薄膜。该变色薄膜以混合纤维素滤膜 为基体，具有良好的柔性。同时，所采用的 RGO 薄 膜导电层的方阻约为 $25.3 \Omega / \square$, 保证了器件良好的 焦耳热性能。在 $6 \mathrm{~V}$ 电压下，薄膜即可实现颜色的多 种变化(如橙红-黄-白或紫红-蓝-白等), 其变色响应 时间约为 $6.3 \mathrm{~s}$, 褪色响应时间约为 $9.2 \mathrm{~s}$ 。

\section{参考文献:}

[1] LEE J, PYO M, LEE S, et al. Hydrochromic conjugated polymers for human sweat pore mapping. Nature Communications, 2014, 5: 3736-3745.

[2] WANG H, SUN Y B, CHEN Q W, et al. Synthesis of carbonencapsulated superparamagnetic colloidal nanoparticles with magnetic-responsive photonic crystal property. Dalton Transactions, 2010, 39(40): 9565-9569.

[3] MA D, SHI G, WANG H, et al. Hierarchical $\mathrm{NiO}$ microflake films with high coloration efficiency, cyclic stability and low power consumption for applications in a complementary electrochromic device. Nanoscale, 2013, 5(11): 4808-4815.

[4] SYUNROKU T. Application of thermochromic materials to films. Kino Zairyo, 1989, 9(5): 39-45.
[5] FAN H, LI K, LI Q, et al. Prepolymerization-assisted fabrication of an ultrathin immobilized layer to realize a semi-embedded wrinkled AgNW network for a smart electrothermal chromatic display and actuator. Journal of Materials Chemistry C, 2017, 5(37): 9778-9785.

[6] HUANG G, LIU L, WANG R, et al. Smart color-changing film with high contrast based on a single-sided conductive fabric. Journal of Materials Chemistry C, 2016, 4(32): 7589-7594.

[7] LAFORGUE A, ROUGET G, DUBOST S, et al. Multifunctional resistive-heating and color-changing monofilaments produced by a single-step coaxial melt-spinning process. ACS Applied Materials \& Interfaces, 2012, 4(6): 3163-3168.

[8] LU X, ZHANG Z, SUN X, et al. Flexible and stretchable chromatic fibers with high sensing reversibility. Chemical Science, 2016, 7(8): 5113-5117.

[9] SHIRAKAWA H, LOUIS E J, MACDIARMID A G, et al. Synthesis of electrically conducting organic polymers: halogen derivatives of polyacetylene, $(\mathrm{CH}) x$. Journal of the Chemical Society, Chemical Communications, 1977(16): 578-580.

[10] FOROUGHI J, SPINKS G M, WALLACE G G. Conducting Polymer Fibers. Handbook of Smart Films, 2015: 31-62.

[11] CHENG Y, WANG R, SUN J, et al. Highly conductive and ultrastretchable electric circuits from covered yarns and silver nanowires. ACS Nano, 2015, 9(4): 3887-3895.

[12] WANG S, CHENG Y, WANG R, et al. Highly thermal conductive copper nanowire composites with ultralow loading: toward applications as thermal interface materials. ACS Applied Materials \& Interfaces, 2014, 6(9): 6481-6486.

[13] GEIM A K, NOVOSELOV K S. The rise of graphene. Nature Materials, 2007, 6(3): 183-191.

[14] WILSON M. Electrons in atomically thin carbon sheets behave like massless particles. Physics Today, 2006, 59(1): 21-23.

[15] WALLACE P R. The band theory of graphite. Physical Review, 1947, 71(9): 622-629.

[16] CHENG Y, WANG R, SUN J, et al. A stretchable and highly sensitive graphene-based fiber for sensing tensile strain, bending, and torsion. Advanced Materials, 2015, 27(45): 7365-7371.

[17] ALOTAIBI F, TUNG T T, NINE M J, et al. Scanning atmospheric plasma for ultrafast reduction of graphene oxide and fabrication of highly conductive graphene films and patterns. Carbon, 2018, 127: 113-121.

[18] LI Q, LI K, FAN H, et al. Reduced graphene oxide functionalized stretchable and multicolor electrothermal chromatic fibers. Journal of Materials Chemistry C, 2017, 5(44): 11448-11453.

[19] HUMMERS JR W S, OFFEMAN R E. Preparation of graphitic oxide. Journal of the American Chemical Society, 1958, 80(6): 1339. 